\title{
Prosody and synchronization in cognitive neuroscience
}

\author{
Franco Orsucci ${ }^{1,2,3^{*}}$, Roberta Petrosino ${ }^{4}$, Giulia Paoloni ${ }^{3}$, Luca Canestri ${ }^{4}$, Elio Conte ${ }^{5,6}$, Mario A Reda $^{4}$ \\ and Mario Fulcheri ${ }^{3}$
}

*Correspondence: f.orsucci@ucl.ac.uk ${ }^{1}$ Division of Psychology and Language Sciences, University College London, London, UK

${ }^{2}$ Cambridgeshire and Peterborough NHS Foundation Trust Cambridge, London, UK

Full list of author information is available at the end of the article

\begin{abstract}
We introduce our methodological study with a short review of the main literature on embodied language, including some recent studies in neuroscience. We investigated this component of natural language using Recurrence Quantification Analysis (RQA). RQA is a relatively new statistical methodology, particularly effective in complex systems. RQA provided a reliable quantitative description of recurrences in text sequences at the orthographic level. In order to provide examples of the potential impact of this methodology, we used RQA to measure structural coupling and synchronization in natural and clinical verbal interactions. Results show the efficacy of this methodology and possible implications.
\end{abstract}

\section{Review}

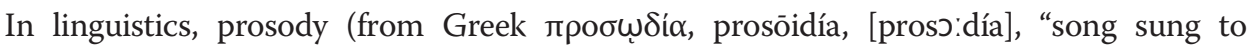
music; pronunciation of syllable") is the rhythm, stress, and intonation of speech. Prosody may reflect various features of the speaker or the utterance: the emotional state of the speaker; the form of the utterance (statement, question, or command); the presence of irony or sarcasm; emphasis, contrast, and focus; or other elements of language that may not be encoded by grammar or choice of vocabulary.

From a neurodynamics perspective, the human brain has a preference for processing emotionally salient stimuli. Emotional prosody can recruit and direct brain processing resources [1]. Besides emotional information, speech prosody can also convey information about linguistic meaning (e.g. determining if a sentence is a statement, a question or a command): prosody and semantics are well connected. Procedural, implicit and emotional knowledge are also embedded in the musical dimension' of language [2].

Words are powerful because, as abstract symbols, they are supposed to transcend personal meanings. They can function at the same time as media for interpersonal communication and individual thinking. They organize and reflect on social and individual experiences. They represent the grounding of logical thinking: symbols (simple as words or abstract as mathematics) enable the detection of relationships and patterns [3-5].

Since the cognitive revolution of the mid-twentieth century, prevailing theories of language in cognitive science, psychology, and linguistics have conceptualized semantics as a complex memory system for categorical knowledge, one that is distinct from 
the system for autobiographical memory [6]. Word meanings were considered to be a-modal abstract entities, independent from the modal systems of perception, action, and emotion [7].

Expanding knowledge on the neural basis of language has posed challenges to the a-modal, disembodied, views of language. An emerging perspective in cognitive neuroscience considers language to be embodied [8]. Embodied cognition supports the perspective that cognitive processes are deeply grounded in perceptual and active engagements with the world [9]. Thinking involved not just as a manipulation of abstract symbols, as it regularly implies a re-enactment of perceptual and motor experiences. Linguistic understanding involves physical experiencing, at least to some degree. This perspective is consistent with the influential work of Lakoff and Johnson [10] on the embodied foundations of language. It also resonates with theories of language developed before the cognitive revolution, including those of Freud [11], conceptualising the close interaction of language and sensorimotor processes [12].

Drawing on diverse research methodologies, studies of embodied language suggest that activation of processes associated with action and perception accompanies the processing of various types of language. In embodied semantics, language is closely integrated with modal systems involved in action, perception, and emotion. Semantic representations are regarded as multimodal and comprising patterns of sensorimotor activation [13]). Barsalou [14] maintains that the embodied semantics model is consistent with known brain dynamics, in contrast to the a-modal view (see also Zwaan and Taylor [15]). Specifically, the principle of Hebbian learning provides a neurological explanation for embodied semantics [16]. In Hebbian learning, neurons that repeatedly fire in sync tend to do so subsequently, so that verbal and sensorimotor processes become "wired together" because neurons that subserve these processes "fired together" during language acquisition (see also Glenberg [17]). Neural synchronization is thoroughly linked to structures and functions of the brain.

\section{Synchronization}

Most of what we have been discussing in terms of coordination, mirroring and reflexivity can be considered, and measured, as synchronization.

In its classical definition, the word synchronization (literally, from ancient Greek, "sharing time") means: adjustment or entrainment of frequencies of periodic oscillators due to a weak interaction [18]. Synchronization is a basic nonlinear phenomenon discovered in interactions between pendulums at the beginning of the modern age of science.

Maturana \& Varela [19] had suggested that synchronisation is a form of structural coupling, which occurs when two systems repeatedly perturb each other. This leads to the development of structural 'fit' between systems. There is a relationship between this process and the emergence of 'appropriate' behaviour from the interplay between interacting systems. Pecora \& Carroll [20] and Ott, Grebogi \& Yorke [21] opened a new way to contemporary research on control and synchronization of complex systems. Maturana [22] highlighted a linguistic and co-evolutionary perspective: "Language is a manner of living together in a flow of coordinations of consensual behaviours or doings that arises in a history of living in the collaboration of doing things together". 
Coordination between conversation partners occurs at multiple levels, including choice of syntactic structure [23]. A number of outstanding questions concerning the origin of this coordination require novel analytic techniques.

In a study on speech and rhythmic behaviour Port et al. [24] found that animals and humans exhibit many kinds of behaviour where frequencies of gestures are related by small integer ratios (like 1:1, 2:1 or 3:1). Many properties like these are found in speech. Dale \& Spivey [25] used this method to explore lexical and syntactic coordination between children and caregivers in conversation. Results indicate that children and caregivers coordinate sequences of syntactic classes, and that this coordination diminishes over development. Similar studies highlight synchronization of eye movements in conversations [26].

\section{Methodology}

We have been investigating synchronization in natural and clinical conversations, finding that it is a complex scaling phenomenon, happening at the same time at the nonverbal, phonetic, syntactic and semantic levels $[27,28]$.

Between the different data analysis methodologies available in dynamical systems theory we found Recurrence Quantification Analysis (RQA) particularly useful for our scopes (Figure 1).

A study in recurrence can be considered as a way to consider order, organization and structure. The study of recurrence of states in nature has a long history in science. Natural processes can have a distinct recurrent behaviour, e.g. periodical repetitions of identical, similar states but also irregular cycles. Moreover, the recurrence of states, is a fundamental property of deterministic order dynamical systems and is typical of nonlinear or chaotic systems. RQA it is proved to be reliable on the relatively short series of data frequent in language analysis and, at the same time, sensitive to changes in the state of the system.

The amount of recurrence is a measure of organisation of a system: if there is no recurrence there is no pattern. Variation in recurring patterns is a measure of change within a given system. A recurrence plot (RP) is a visualisation in a graph of a square matrix, in which points and lines correspond to times when states of a system recur, replicate. More technically, the RP reveals all the times when the phase space trajectory of the dynamical system visits roughly the same area in the phase space. Such recurrence of a state at time $i$ at a different time $j$ is marked within a two-dimensional squared matrix with ones and zeros (black and white) dots in the plot, where both axes
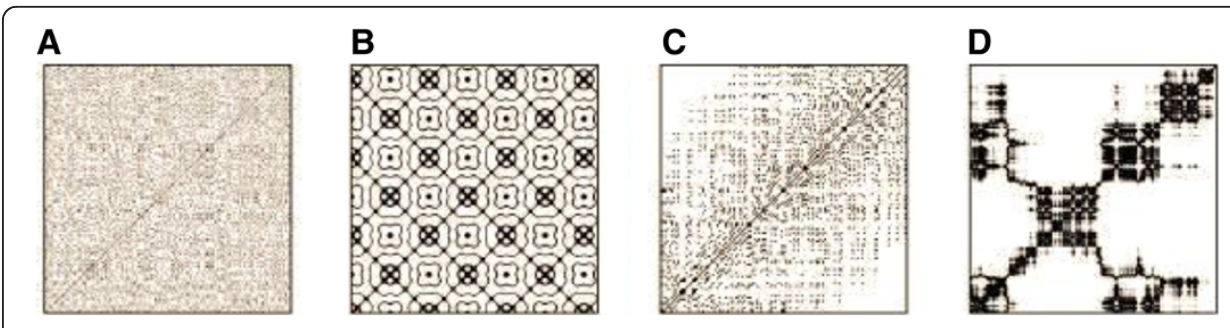

Figure 1 Characteristic typology of recurrence plots: (A) homogeneous (uniformly distributed noise), (B) periodic (super-positioned harmonic oscillations), (C) drift (logistic map corrupted with a linearly increasing term) and (D) disrupted (Brownian motion). 
are time axes. If the plot is in color, depending on the software used, color highlights a different density of recurrence points. The study of RPs from paradigmatic systems gives a good introduction into characteristic typology and texture of system organisations. However, their quantification offers a more objective way for the investigation of the considered system. With quantification, RPs have become more and more popular within a growing group of scientists who use them for data analysis (for example, a search with Google Scholar reveals over 1200 published works about Recurrence Plots over the last 5 years).

Recurrence Plots (RP) were first introduced in physics by Eckmann et al. [29]. Later, Webber, Zbilut and Giuliani $[30,31]$ enhanced this technique by defining nonlinear variables diagnostically useful in the quantitative assessment of RPs, so developing RQA. Recurrence Quantification Analysis (RQA) is a method of nonlinear data analysis which quantifies the number and duration of recurrences of a dynamical system as they are represented in its state space trajectory. RQA has been used in different fields, ranging from molecular dynamics to physiology and bioinformatics. In order to be processed by RQA, the original series must be transformed into its embedding matrix by the agency of the method of delays transforming the original $n$ elements column vector correspondent to the symbol series into a $p$-dimensional matrix having as columns the original $X_{n}$ series plus its lagged copies $X_{n+1}, X_{n+2} ; \ldots ; X_{n+p-1}$, the value $p$ being the embedding dimension.

The application of RQA is based on the calculation of the Euclidean distance between all the pairs of rows of the embedding matrix. If the distance between two generic rows (i.e., windows of predefined length along the sequence) falls below the radius, we obtain a recurrence.

The concept of recurrence is straightforward: for any ordered series (time or spatial), a recurrence is a point which repeats itself. In this respect, the statistical literature points out that recurrences are the most basic of relations shaping a given system, since they are strictly local and independent of any mathematical assumption regarding the system itself. Furthermore, it is worth stressing that recurrences computation requires no transformation of the data and can be used for both linear and nonlinear systems. The concept of a recurrence can be expressed as follows: given a reference point, $X_{0}$, and a ball of radius $r$, point $X$ is said to recur (with reference to $X_{0}$ ) if $\left\{X:\left\|X-X_{0}\right\| \leq r\right\}$.

In the case of a time series, i.e., of a system occupying in different times different positions along a trajectory in a suitable state space, the recurrences correspond to the time points where the system passes nearby to already visited states. In the case of texts, time corresponds to the order in which the different letters appear: the recurrences are patches, with a length equal to the embedding dimension, sharing their letter profile with other patches along the chain.

Depending on the embedding dimension these patches will correspond to specific words or phrases.

The number and relative positions of recurrences are expressed by recurrence plots (RP), that are symmetrical $N \cdot N$ arrays in which a point is placed at $(i, j)$ whenever a point $X_{i}$ on the trajectory is close to another point $X_{j}$. The closeness between $X_{i}$ and $X_{j}$ is expressed by calculating the Euclidian distance between these two normed vectors, i.e., by subtracting one from the other obtaining the expression $\left\|X_{i}-X_{j}\right\| \leq r$, where $r$ is a fixed radius. If the distance falls within this radius, the two vectors are considered to be 
recurrent, and graphically this can be indicated by a dot. In the case of alphabetical strings, the radius is constrained to be zero and the similarity collapses to the identity of motifs along the text.

Thus, recurrence plots correspond to the distance matrix between the different epochs (rows of the embedding matrix) filtered, by the action of the radius, to a binary 0/1 matrix having a 1 (dot) for distances falling below the radius and a 0 for distances greater than the radius.

An important feature of such matrixes is the existence of short line segments parallel to the main diagonal, which correspond to sequences $(i, j),(i+1, j+1), \ldots,(i+k, j+k)$ such that the fragment $X(j), X(j+1), X(j+k)$ is close to $X(i), X(i+1), \ldots, X(i+k)$. The absence of such patterns suggests randomness. For texts these deterministic lines correspond to contiguous patches of letters repeating

themselves in different portions of the text. They can represent identical words or phrases appearing in different moments of a speech. The 'line' is a measurement parameter which states the minimum number of adjacent recurrent points required to define a deterministic line. We set our line to 2, that corresponds to say that we need two contiguous recurrent patches of 3 to get a deterministic line.

The quantification of recurrences is obtained by many different 'counts' of recurrences disposition on the matrix. Here we will limit ourselves to the two most basic descriptors:

- Recurrence (REC): percentage of recurrence points in the recurrence plot.

- Determinism (DET): percentage of recurrent points which form diagonal lines.

Basic RQA refers to the autocorrelation structure of a given series, Zbilut et al. [32] demonstrated the applicability of the method to the quantification of the 'crosscorrelation' structure of different series; this possibility was further exploited and refined by Marwan et al. [33] who developed the concept of cross-recurrence quantification analysis (CRQA). Conte et al. highlighted the possible usage of RQA to detect singularities [34].

The basic formalism is identical, but in this case the $\mathrm{X}$ and $\mathrm{Y}$ axis of the recurrence plot are no more coincident but refer to the two different signals to be compared. This obviously eliminates the recurrent main diagonal and shifts the meaning of the dots to 'repetitions' inside the same text to concordances scored between different texts.

The average length of the diagonal lines and Max Line estimation is also of valuable importance since the inverse of Max Line, relates to Kolmogorov-Sinai entropy and thus the sum of positive Lyapunov exponents. The Entr, (entropy) is a measure of the Shannon entropy and directly relates estimation of the complexity of the system. The Trend is the pailing of the RP towards its edges. Diagonal structures in RP relate chaos order transitions. N. Marwan [33] introduced also the so called $\boldsymbol{L A M}$ variable. It estimates laminarity that is to say the percentage of recurrence points forming vertical lines and the Trapping Time estimating the average length of the vertical lines. Laminarity relates to chaos-chaos transitions.

estimates laminarity that is to say the percentage of recurrence points forming vertical lines and the Trapping Time estimating the average length of the vertical lines. Laminarity relates to chaos-chaos transitions. 
We applied the RQA methodology to prosodic data as represented in orthography. In order to reach a robust quantitative measure of prosody, we decided to focus on the orthography of written texts, including transcribed verbal communications.

Orthography generally refers to spelling; that is, the relationship between phonemes and graphemes in a given language. Orthography defines the set of symbols (graphemes and diacritics) used in a language, and the rules about how to write these symbols. Most natural languages developed as oral languages, and writing systems have usually been crafted or adapted afterwards as representations of the spoken language. An orthography may be described as efficient if it has just one grapheme per phoneme (distinctive speech sound) and vice versa. An orthography may also have varying degrees of efficiency for reading or writing. Our studies have been focussing on prosody as it is reflected in orthography. Knowledge of the informational structures of orthography has a consistent and robust scientific background starting with the classical studies in information theory by Claude Shannon [35].

In a mentioned preliminary work [27] we had investigated the informational structure of written texts (including speech transcriptions) using Recurrence Quantification Analysis (RQA). As mentioned, orthography is a combination of conventions, and its relations with phonetics can be different for every single language, but we found a robust confirmation that RQA it is a reliable measure for it, across different languages:

1) relative invariance with respect to the original language of texts;

2) consistency with known and recognizable structures;

3) maximal dependence on dynamical (order dependent) features of texts;

4) relative independence on statistical (order independent) features. Experimental evidence suggests that Recurrence Quantification Analysis (RQA) satisfies these requirements. As RQA is based on a plain matching of pairs, it is not affected by coding biases: some of the software used for Recurrence Quantification Analysis can measure written texts, and no preliminary coding is needed (Figure 2).

\section{Results}

Once established the reliability and robustness of this methodology for orthographic studies, we used RQA to measure coupling and synchronization of different subjects during natural and clinical conversations.

We measured series of text samples derived from transcriptions of natural, spontaneous, conversations (NAT) and clinical conversations (CLIN). While the first ones were supposed to evolve following their natural co-evolutionary dynamics; the second ones were supposed to be finalized by therapists towards controlled and partially pre-defined dynamics (therapeutic goals). The clinical examples we are presenting in this work are derived from three different contexts: a psychiatric assessment; a cognitive psychotherapy session and a psychodynamic psychotherapy session. All of them highlight interesting patterns of prosodic organization, synchronization, co-evolution and co-construction. Sample A, NAT, as represented in Figure 3-A, is a natural dialogue between two friends: they are talking about some general topic such as "the meaning of life". While in the beginning of conversation they seem quite well synchronized, as they approach the end of their conversation and separation they tend to decouple. 


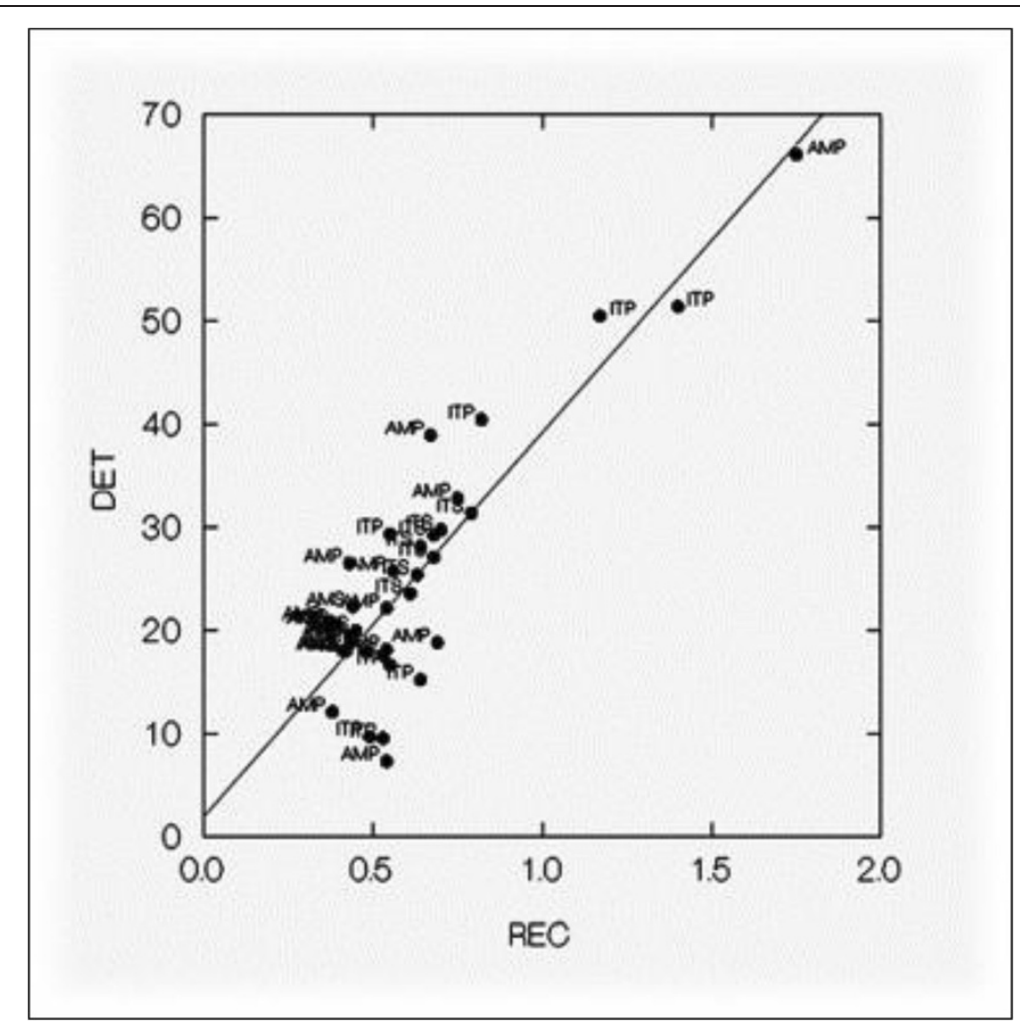

Figure 2 Plot of structure distribution of all described linguistic samples: a combined analysis of American (AMP) and Italian poems (ITP); American (AMS) and Italian speech transcriptions (ITS).

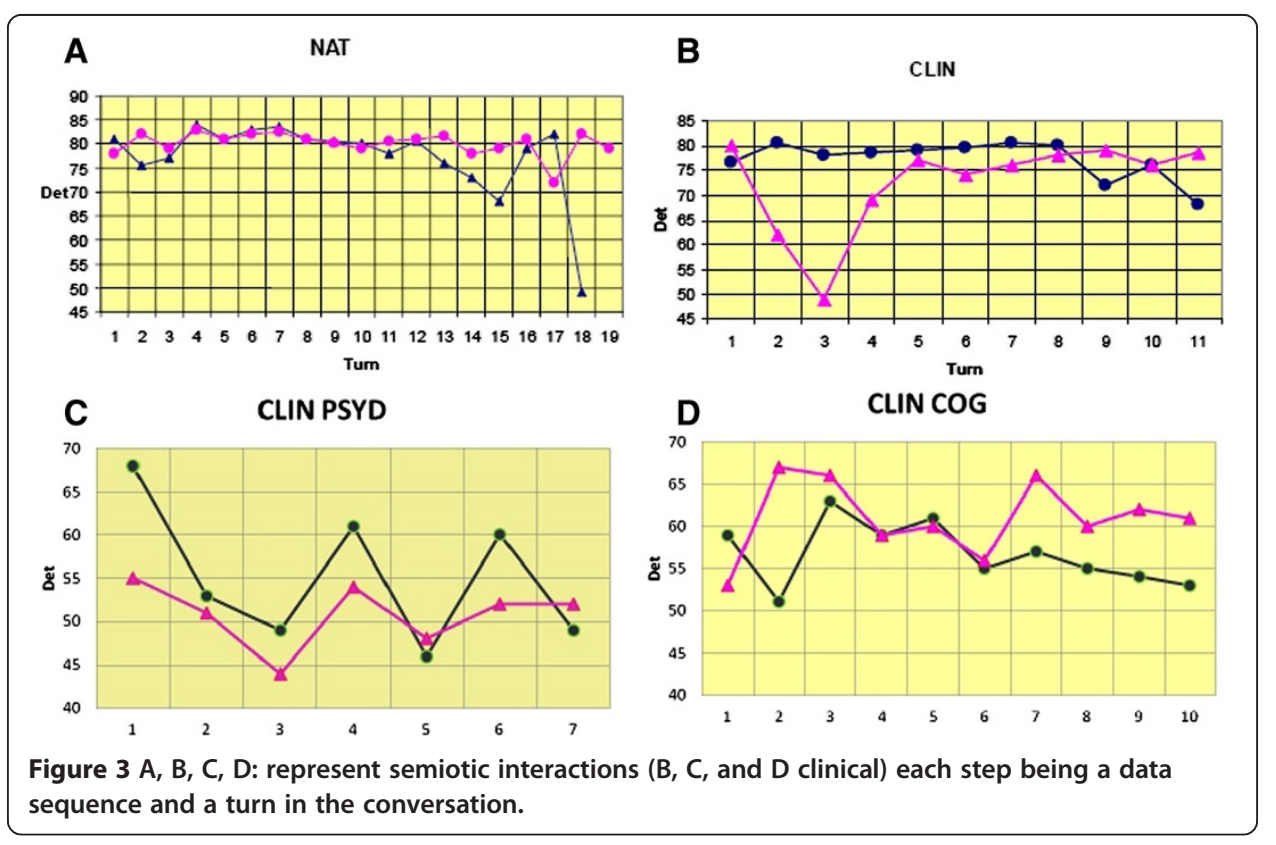


Sample B, CLIN, as represented in Figure 3-B, is clinical dialogue between a patient and a psychiatrist: this is a first interview. They start in sync, but soon de-sync, to find slowly a new sync including some crossing.

Sample C, CLIN PSYD, as represented in Figure 3-C, is a psychodynamic psychotherapy session. There is evidence of medium strength coupling and one crossing.

Sample D, CLIN COG, as represented in Figure 3-D, is a cognitive psychotherapy session with evidence of double crossing and strong coupling at mid-session, soon followed by decoupling.

Embedding 3 was the most reliable metrics to highlight synchronization. Higher embedding measures would have highlighted correlations at the word level (embedding 5 to 8 ) or at the phoneme level (embedding 1 or 2). These data were produced at embedding 3 (this means we considered units of three letters). This means that synchronization is more active at the meso level (slightly higher than single letters, phonemes, information). This level implies resonances in rhythms and morphemes. A level lower than narrative macro structures but higher than micro structures (letters, phonemes). This probably might mean that coupling and synchronization in conversation (semiotic interaction) start in non-aware ways, before some self awareness implied in higher narrative structures is established.

\section{Conclusions}

Language is the fabric of human mind and a scientific study of language can provide precious understandings of neuro-cognitive processes. The study of language is differentiated on its complex levels of structuring: from informational microscopic systems, to morphological mesoscopic patterns, to semantic and narrative macroscopic pathways. The methodology we presented is mostly focused on micro and meso structures but, as language is like a complex living and changing organism, just as our minds are, interactions are continuously intermingling.

The dynamical systems' approach to language is a defining trend in linguistics and semiotics [36-38]. Following this theory, lexicon consists of regions of state space within a semiotic system. Grammar consists of the dynamics (attractors and repellers) which constrain dynamics in that space. Representations are highly context-sensitive, continuously varied and probabilistic. They are regions in a state-space.

Our research can be considered complimentary with study by Shockley et al. [39], in which interpersonal coordination during conversation was based on recurrence strategies, to evaluate the shared activity between two postural time series in a reconstructed phase space.

Animals are dynamical, self-organizing, systems in which contents and representations emerge from the systemic tendency of open, non-equilibrium systems to form patterns. In the case of actor-perceivers, as Kelso [40] notes, intelligent behaviors emerge in the absence of agents directing those behaviors.

Individuals and their environments are, properly considered, coupled dynamical systems, with coupling being both informational and mechanical in nature. Interactions between the systems give rise to a behavioural layout having attractors corresponding to stable behaviors that achieve intended goals and bifurcations that lead to transitions between behaviors. 
Being in a common frame of reference with another may be the basis for individuals forming a comfortable, smoothly operating social unit, something that should be indicated by feelings of "harmony", or greater perceptual and cognitive fluency. This might be considered the common cognitive ground for the specialised linguistic interaction developed in psychotherapy as a therapeutic alliance.

Language use is inherently cooperative in nature. This notion is evidenced by a variety of studies that have demonstrated the spontaneous convergence in speaking patterns in social settings. For example, verbal interactions among individuals demonstrate convergence in dialect [41], speaking rate [42], vocal intensity [43], and pausing frequency $[44,45]$. These tendencies to coordinate speech patterns likely have a social, or cooperative, motivation [46]. The tendency to coordinate and imitate persists into adulthood. For example, adults imitate facial expressions [47]. Interlocutors do not limit their convergences to speech. They are said to move in synchrony with each other's speech rhythms [48] and to match one another's postures $[49,50]$ in the absence of an intention to entrain. For example, LaFrance [51] demonstrated that listeners to a speaker whom they find engaging tend to mirror the speaker's postures.

Listeners are also reported to move in time with the rhythms of a speaker's speech (exhibiting "interactional synchrony"; [52,53]).

Shockley, et al. [54], evaluated the degree of shared postural sway activity in the context of a cooperative verbal task. The stable organization and patterning of joint action emerges from informational couplings that exist between individuals and between individuals and the environment. In such a paradigm, therefore, the implicit commitment to act as a "plural subject" of action, that is, to choose to cooperate, is something that emerges without prior planning or a priori expectations, in response to individual, environmental, and social constraints.

When people are co-present and in communication the speech rhythms and articulatory processes of two people become coordinated in a natural conversation and postural activity. Infants' sensitivity for the rhythms and melodic or prosodic features of adult vocalisations, i. e. their adaptations for 'Communicative Musicality' [55] has been studied, with particular attention to responses to infant-directed forms of maternal vocalisations (speech, song and sound games) in which rhythmic expression and affective quality are enhanced.

Our findings in the synchronization of conversation dynamics can be relevant for the general issue of structural coupling of psychobiological organizations.

Competing interests

The authors declare that they have no competing interests.

Authors' contributions

FO designed the methodology, the study and provided time series; RP provided and analysed time series;

GP provided a systematic review; LC provided time series; MAR improved the methodology; MF improved the methodology. All authors read and approved the final manuscript.

Author details

${ }^{1}$ Division of Psychology and Language Sciences, University College London, London, UK. ${ }^{2}$ Cambridgeshire and Peterborough NHS Foundation Trust Cambridge, London, UK. ${ }^{3}$ Clinical Psychology Laboratory, University of ChietiPescara, Chieti-Pescara, Italy. ${ }^{4}$ Department of Behavioral and Neurological Sciences, University of Siena, Siena, Italy. ${ }^{5}$ Department of Neurosciences, University of Bari Aldo Moro, Bari, Italy. ${ }^{6}$ School of Advanced International Studies on Applied Theoretical and non Linear Methodologies of Physics, Bari, Italy. 


\section{References}

1. Wiethoff S, Wildgruber D, Becker H, Anders S, Herbert C, Grodd W, Ethofer T: Cerebral processing of emotional prosody - Influence of acoustic parameters and arousal. Neuroimage 2008, 39:885-893.

2. Mancia M: Implicit memory and early unrepressed unconscious: Their role in the therapeutic process (How the neurosciences can contribute to psychoanalysis). Int J Psychoanal 2006, 87:83-103.

3. Bucci W: Psychoanalysis and cognitive science: A multiple code theory. New York: Guilford Press; 1997.

4. Smith LB, Gasser M: The development of embodied cognition: six lessons from babies. Artif Life 2005, 11:13-30.

5. Olds DD: A semiotic model of mind. J Am Psychoanal Assoc 2000, 48:497-529.

6. Fodor J: The Language of Thought. Cambridge: Harvard University Press; 1975.

7. Gallese V, Lakoff $\mathrm{G}$ : The brain's concepts: The role of the sensory-motorsystem in conceptual knowledge. Cogn Neuropsychol 2005, 22:455-479.

8. Fischer MH, Rolf AZ: Embodied language: a review of the role of the motor system in language comprehension. Q J Exp Psychol 2008, 61(6):825-850.

9. Wilson M: Six views of embodied cognition. Psychon Bull Rev 2002, 9:625-636.

10. Lakoff G, Johnson M: Metaphors We Live By. Chicago: University of Chicago Press; 1980

11. Freud S: On Aphasia. A Critical Study. In Cerebral processing of emotional prosody - Influence of acoustic parameters and arousal. New York: International Universities Press, (1891); 1953.

12. Martin A: The representation of object concepts in the brain. Annu Rev Psychol 2007, 58:25-45.

13. Aziz-Zadeh L, Wilson SM, Rizzolatti G, lacoboni M: Congruent embodied representations for visually presented actions and linguistic phrases describing actions. Curr Biol 2006, 16:1818-1823.

14. Barsalou LA: Grounded cognition. Annu Rev Psychol 2008, 59:617-645.

15. Zwaan RA, Taylor LJ: Seeing, acting, understanding: Motor resonance in language comprehension. J Exp Psychol Gen 2006, 135:1-1 (1).

16. Pulvermüller F: Brain mechanisms linking language and action. Nature Reviews of Neuroscience 2005, 6:576-582.

17. Glenberg AM, Sato M, Cattaneo L, Riggio L, Palumbo D, Buccino G: Processing abstract language modulates motor system activity. Q J Exp Psychol 2008, 61:905-919.

18. Strogatz SH: Sync, The Emerging Science of Spontaneous Order. New York: Hyperion; 2003.

19. Maturana HR, Varela FJ: Autopoiesis and cognition, the realization of the living. Dordrecht, Holland: Reidel Pub. Co; 1980.

20. Pecora LM, Carroll TL: Synchronization in chaotic systems. Phys Rev Lett 1990, 64:821-824

21. Ott E, Gerbogi C, Yorke JA: Controlling Chaos. Phys Rev Lett 1990, 64(11):1196-1199.

22. Maturana H: Autopoiesis, structural coupling and cognition: a history of these and other notions in the biology of cognition. Instituto Matriztico 2002, 9(3-4):3-4. Internet publication.

23. Branigan HP, Pickering MJ, Cleland AA: Syntactic coordination in dialogue. Cognition 2000, 75:B13-25.

24. Port R, Tajima K, Cummins F: Speech and rhythmic behavior. In The Nonlinear Analysis of Developmental Processes. Edited by Savelsburgh GJP, van der Maas H, van Geert PCL. Amsterdam: Elsevier; 1999.

25. Dale R, Spivey MJ: Unraveling the dyad: Using recurrence analysis to explore patterns of syntactic coordination between children and caregivers in conversation. Lang Learn 2006, 56:391-430.

26. Richardson DC, Dale R: Looking to understand: The coupling between speakers' and listeners' eye movements and its relationship to discourse comprehension. Cogn Sci 2005, 29:39-54.

27. Orsucci F, Walters K, Giuliani A, Webber C Jr, Zbilut J: Orthographic Structuring of Human Speech and Texts: Linguistic Application of Recurrence Quantification Analysis. International Journal of Chaos Theory and Applications 1999, 4-2:80-88.

28. Orsucci FF: The paradigm of complexity in clinical neurocognitive science. Neuroscientist 2006, 12(5):390

29. Eckmann JP, Kamphorst SO, Ruelle D: Recurrence plots of dynamical systems. Europhys Lett 1987, 4:973-977.

30. Zbilut JP, Webber CL: Embeddings and delays as derived from quantification of recurrence plots. Physics letters A 1992, 171(3):199-203.

31. Giuliani A, Manetti C: Recurrence quantification analysis as a tool for characterization of molecular dynamics simulations. Phys Rev E 1996, 53:6336.

32. Zbilut JP, Giuliani A, Webber CL: Detecting deterministic signals in exceptionally noisy environments using cross-recurrence quantification. Phys Lett A 1998, 246(1):122-128.

33. Marwan N, Romano MC, Thiel M, Kurths J: Recurrence plots for the analysis of complex systems. Phys Rep 2007, 438(5-6):237-329.

34. Conte E, Vena A, Federici A, Giuliani R, Zbilut JP: A brief note on possible detection of physiological singularities in respiratory dynamics by recurrence quantification analysis of lung sounds. Chaos, Solitons Fractals 2004, 21(4):869-877.

35. Shannon CE: Prediction and Entropy of Printed English. Bell System Techn J 1951, 30:50.

36. Orsucci F: Changing Mind. Singapore: Transitions in natural and artificial environments World Scientific Publishing; 2002.

37. Bates E: The emergence of symbols, cognition and communication in infancy. New York: Academic Press; 1979.

38. Elman JL: Rethinking innateness, a connectionist perspective on development. Cambridge, Mass: MIT Press; 1996.

39. Shockley K, Santana MV, Fowler CA: Mutual interpersonal postural constraints are involved in cooperative conversation. J Exp Psychol Hum Percept Perform 2003, 29:326-332.

40. Kelso JAS: Dynamic patterns: The self-organization of brain and behavior. Cambridge, MA: MIT Press; 1995.

41. Giles H: Accent mobility: A model and some data. Anthropological Linguistics 1973, 15:87-105.

42. Street RL: Speech convergence and speech evaluation in fact-finding interviews. Human Communication Research Jr. 1984, 11:139-169.

43. Natale M: Convergence of mean vocal intensity in dyadic communication as a function of social desirability. J Pers Soc Psychol 1975, 32:790-804.

44. Capella J, Planalp S: Talk and silence sequences in informal conversations, III: Interspeaker influence. Human Communications Research 1981, 7:117-132. 
45. Giles H, Coupland N, Coupland J: Accommodation theory: Communication, context, and consequence. In Contexts of accommodation: Developments in applied sociolinguistics. Edited by Giles H. New York: Cambridge University Press; 1991:1-68.

46. Bourhis RY, Giles H: The language of intergroup distinctiveness. In Language, Ethnicity and Intergroup Relations. Edited by Giles H. London: Academic Press; 1977:119-135.

47. McHugo G, Lanzetta J, Sullivan D, Masters R, Englis B: Emotional reactions to a political leader's expressive displays. J Pers Soc Psychol 1985, 49:1513-1529.

48. Condon W: An analysis of behavioral organization. Sign Language Studies 1976, 13:285-318.

49. Condon WS, Ogston WD: Sound film analysis of normal and pathological behavior patterns. J Nerv Ment Dis 1966, 143:338-347.

50. Kendon A: Movement coordination in social interaction: Some examples. Acta Psychol 1970, 32:1-25.

51. LaFrance M: Posture mirroring and rapport. In Interaction rhythms: Periodicity in communicative behavior. Edited by Davis M. New York: Human Sciences Press; 1982.

52. Condon W, Ogston W: Speech and body motion synchrony of the speakerhearer. In The Perception of Language. Edited by Horton D, Jenkins J. Columbus, OH: Charles E. Merrill; 1971:150-184.

53. Newtson D: The perception and coupling of behavior waves. In Dynamical systems in social psychology. Edited by Vallacher R, Nowak A. San Diego CA: Academic Press; 1994:139-167. San.

54. Shockley KD, Baker AA, Richardson MJ, Fowler CA: Articulatory constraints on interpersonal postural coordination. Journal of Experimental Psychology: Human Perception and Performance; 2010.

55. Malloch S: Mothers and infants and communicative musicality. Musicae Scientie 1999, 3(1 suppl):29-57.

doi:10.1140/epjnbp13

Cite this article as: Orsucci et al:: Prosody and synchronization in cognitive neuroscience. EPJ Nonlinear Biomedical

Physics 2013 1:6.

\section{Submit your manuscript to a SpringerOpen ${ }^{\circ}$ journal and benefit from:}

- Convenient online submission

- Rigorous peer review

- Immediate publication on acceptance

- Open access: articles freely available online

- High visibility within the field

- Retaining the copyright to your article

Submit your next manuscript at $>$ springeropen.com 